\title{
Editorial: Viscoelasticity: From Individual Cell Behavior to Collective Tissue Remodeling
}

\author{
Ivana D. Pajic-Lijakovic ${ }^{1 *}$, Karine Guevorkian ${ }^{2}$, Elias H. Barriga ${ }^{3}$ and Jose J. Muñoz ${ }^{4,5,6}$ \\ ${ }^{1}$ Department of Chemical Engineering, Faculty of Technology and Metallurgy, University of Belgrade, Belgrade, Serbia, ${ }^{2}$ Institut \\ Curie, Université PSL, Sorbonne Université, CNRS UMR168, Laboratoire Physico Chimie Curie, Paris, France, ${ }^{3}$ Mechanisms of \\ Morphogenesis Laboratory, Gulbenkian Institute of Science (IGC), Oeiras, Portugal, ${ }^{4}$ Universitat Politecnica de Catalunya, \\ Barcelona, Spain, ${ }^{5}$ Centre Internacional de Mètodes Numèrics en Enginyeria (CIMNE), Barcelona, Spain, ${ }^{6}$ Institut de \\ Matemàtiques de la UPC-BarcelonaTech (IMTech), Barcelona, Spain
}

Keywords: viscoelasticity of multicellular systems, collective cell rearrangement, multi scale mathematical modeling, elastography, cell jamming state transition, active elastic turbulence

\section{Editorial on the Research Topic}

\section{OPEN ACCESS}

Edited and reviewed by: Ralf Metzler, University of Potsdam, Germany

*Correspondence: Ivana D. Pajic-Lijakovic iva@tmf.bg.ac.rs

Specialty section:

This article was submitted to Biophysics,

a section of the journal

Frontiers in Physics

Received: 09 September 2021 Accepted: 15 September 2021 Published: 29 September 2021

Citation:

Pajic-Lijakovic ID, Guevorkian K, Barriga EH and Muñoz JJ (2021)

Editorial: Viscoelasticity: From Individual Cell Behavior to Collective

Tissue Remodeling.

Front. Phys. 9:773096.

doi: 10.3389/fphy.2021.773096

\section{Viscoelasticity: From Individual Cell Behavior to Collective Tissue Remodeling}

This issue gathers exciting multi-disciplinary work relating viscoelasticity and collective cell remodeling within various biological processes such as morphogenesis, tumorigenesis, and wound healing. Viscoelasticity is influenced by energy transfer and dissipation during cell rearrangement at various time and space scales. Cumulative structural changes at a subcellular level have effects on viscoelasticity at a supracellular level. Established configurations of migrating cells and the rate of their change, which significantly regulate viscoelasticity at a supracellular level, have the impact on the cohesiveness inhomogeneity and various mechanical and biochemical processes at a subcellular level. This Research Topic aims to connect the macroscopic viscoelastic parameters with the individual and collective cell response. Consideration of biochemical, biophysical and bio-mechanical aspects responsible for tissue remodeling, intercalation, and migration were discussed on various multicellular systems under in vivo and in vitro conditions.

Thus in this Research Topic we aim to provide a state-of-the-art view about the current knowledge related to viscoelasticity caused by collective cell remodeling and adhesive contractile properties, covering a plethora of phenomena such as: 1) single cell response under stretched monolayers modeled with an improved Vertex model, 2) adhesion percolation within a tissue as an important factor which influences its viscoelasticity, 3) the active turbulence caused by collective cell migration accompanied with the generation of mechanical waves, 4) cell jamming state transitions, and 5) viscoelastic response characterization in liver diseases. Alternative techniques to measure and control cell rearrangement under various experimental conditions are also considered, including atomic force microscopy measurements and various elastography techniques. 
This Research Topic provides an overview of the current understanding of various: biological, biochemical, biophysical and mechanical aspects of cell remodeling. The inter-relation between cell remodeling and tissue viscoelasticity was discussed by emphasizing the relevant rheological parameters, the way of their measurement under in vivo/ in vitro conditions, and the strategy of multi-scale constitutive modeling.

\section{AUTHOR CONTRIBUTIONS}

All authors listed have made a substantial, direct, and intellectual contribution to the work and approved it for publication.
Conflict of Interest: The authors declare that the research was conducted in the absence of any commercial or financial relationships that could be construed as a potential conflict of interest.

Publisher's Note: All claims expressed in this article are solely those of the authors and do not necessarily represent those of their affiliated organizations, or those of the publisher, the editors and the reviewers. Any product that may be evaluated in this article, or claim that may be made by its manufacturer, is not guaranteed or endorsed by the publisher.

Copyright $\odot 2021$ Pajic-Lijakovic, Guevorkian, Barriga and Muñoz. This is an openaccess article distributed under the terms of the Creative Commons Attribution License (CC BY). The use, distribution or reproduction in other forums is permitted, provided the original author(s) and the copyright owner(s) are credited and that the original publication in this journal is cited, in accordance with accepted academic practice. No use, distribution or reproduction is permitted which does not comply with these terms. 